\title{
评 述＼cjkstart离子液体与绿色工程专刊
}

\section{离子液体在结构相似物分离中的进展}

\author{
金文涁，李雪楠，张依，杨启炜，邢华斌"，任其龙
}

生物质化工教育部重点实验室, 浙江大学化学工程与生物工程学院, 杭州 310027

*通讯作者, E-mail: xinghb@zju.edu.cn

收稿日期: 2016-06-04; 接受日期: 2016-07-19; 网络版发表日期: 2016-11-25

国家自然科学基金(编号: $21222601,21476192,21436010$ )和国家万人计划青年拔尖人才资助项目

\begin{abstract}
摘要离子液体作为新型的分离介质近年来受到研究者的广泛关注. 特别是离子液体具有多重溶剂化能力和 可调的结构和性质, 可实现化合物微小结构差异的专一性识别, 在结构相似物分离中表现出优异的分离选择性. 离子液体用于结构相似物的分离正成为分离科学研究的前沿领域. 本文综述了离子液体的结构特征及其与溶质 分子的相互作用规律, 归纳了离子液体在天然活性物质、有机化合物和低碳烃等结构相似物的分离和纯化方面 取得的最新进展，探讨了离子液体结构相似物分离研究存在的问题和未来发展方向.
\end{abstract}

关键词离子液体, 分离, 生物活性物质, 低碳烃, 结构相似物

\section{1 引言}

理想的分离介质需具备以下特性：对溶质具有 良好的溶解能力, 可以在分离过程中带来较大的分 配系数和较高的处理能力; 与不同溶质之间具有较 强的选择性作用能力，能选择性识别目标溶质与杂 质间的性质差异，达到较高的分离选择性; 分离过程 中不易流失，降低与其他相之间的交叉污染; 分离结 束后易于再生, 可循环使用等. 水和常规有机溶剂等 分离介质往往难以兼具上述性能. 离子液体 (ionic liquids, ILs)作为一种新型溶剂, 是完全由阴、阳离子 组成在室温或接近室温条件下呈液态的盐类. 与传 统分子溶剂相比, 离子液体独特的结构使之具有独 特的物理化学性质, 如蒸气压低、难挥发、对热稳定 和内聚能高、不易燃、溶解能力好、液程和电化学窗
口较宽、易形成液-液两相 ${ }^{[1,2]}$, 这些特性不仅使离子 液体可作为环境友好的溶剂替代传统挥发性有机溶 剂, 更为清洁化的化学化工过程中提供了创新的源 头 ${ }^{[3,4]}$. 近年来, 离子液体已经在萃取分离、催化、材 料、生物质能源、环境、电化学、石油化工等诸多领 域展现了良好的应用前景 ${ }^{\left[{ }^{[} \sim 8\right]}$.

离子液体的性质可以通过阴阳离子结构改变进 行调节 ${ }^{[9]}$ ，是一类“可设计溶剂”，通过对离子液体的 阴阳离子的设计可调节其对无机物、水、有机物、聚 合物等的溶解性, 精细调控离子液体与分离对象的 相互作用方式及强度，实现化合物结构微小差异的 分子识别. 自 1998 年 Rogers 等 ${ }^{[10]}$ 首先报道了用离子 液体从水中萃取分离酚类衍生物以来，基于离子液 体的分离方法已被广泛用于有机小分子化合物 ${ }^{[11,12]}$ 、 金属离子和稀土元素 ${ }^{[13,14]}$ 、天然活性物质 ${ }^{[15,16]}$ 、气体

引用格式: 金文彬, 李雪楠, 张依, 杨启炜, 邢华斌, 任其龙. 离子液体在结构相似物分离中的进展. 中国科学: 化学, 2016, 46: 1251-1263 Jin W, Li X, Zhang Y, Yang Q, Xing H, Ren Q. Separation of structurally-related compounds with ionic liquids. Sci Sin Chim, 2016, 46: 1251-1263, doi: $10.1360 /$ N032016-00121 
分子 ${ }^{[17,18]}$ 等分离. 本文简要评述了近几年来以离子 液体为介质分离结构相似物方面的进展, 分析了离 子液体与溶质分子间的相互作用以及离子液体在实 现绿色分离过程中的作用, 探讨了存在的主要问题, 并对其应用前景进行了展望.

\section{2 离子液体的性质及其与溶质分子的相互 作用}

离子液体是完全由阴、阳离子组成的离子型化合 物, 存在着特有的微观静电场和分子环境, 在结构和 性质上与传统的分子溶剂存在明显区别. 离子液体结 构中往往同时具有疏水、不饱和键、氢键供体、氢键 受体、静电等多种结构片段, 因此离子液体可与溶质 之间发生疏水、氢键、静电、 $\pi-\pi$ 等多种相互作用, 使 离子液体具有传统分子溶剂不能比拟的优势 ${ }^{[19]}$. 因此, 明确离子液体与溶质分子之间的相互作用, 对于离子 液体分子设计及应用具有重要意义.

溶剂极性经验参数的研究结果表明, 大部分的离 子液体拥有与短链醇类相似的极性 ${ }^{[20]}$. 但是单纯使用 极性来阐述离子液体的特性, 并不能解释为何不同组 合的阴阳离子所构成的离子液体, 会产生独特的行 为 ${ }^{[20]}$. Armstrong 等 ${ }^{[21]}$ 报道采用 Abraham 提出的“溶剂 化参数模型” (solvation parameter model), 来表示离子 液体与溶质探针分子之间的相互作用. 通过测定 17 种 离子液体与多种探针分子的溶剂化参数, 揭示了离子 液体与溶质分子之间存在着多重溶剂化作用. Welton 等 ${ }^{[22]}$ 进一步采用溶剂化方法表征了离子液体中的溶剂 化参数, 其结果表明, 离子液体作为一种极性溶剂, 与溶质存在多种相互作用, 在适当条件下, 既可以作 为氢键供体也可以成为氢键受体. $\mathrm{Xu}$ 等 ${ }^{[23]}$ 将溶剂化作 用与离子液体结构性质关联溶解度, 利用真实溶剂似 导体屏蔽模型(conductor-like screening model for real solvents, COSMO-RS) 方法预测了黄酮类物质在离子 液体中的溶解度, 其计算结果表明, 离子液体与溶质 之间具有多重相互作用, 而其中氢键碱性对溶解度占 有主导性的影响. 氢键和氢键碱性一直是离子液体物 化性质研究中的重点. 离子液体与溶质之间的氢键酸 碱相互作用在离子液体的应用中起到重要的作用, 对 于很多化合物, 氢键碱性的提高往往能够提高溶解度 或带来较好的分离效率. Rogers 等 ${ }^{[24]}$ 发现, 1-丁基-3-甲 基咪唑氯盐([Bmim] Cl)离子液体在微波辅助下可以有
效溶解纤维素等生物大分子, 而离子液体较强的氢键 作用是其溶解的关键. 此外, 已有越来越多的研究证 明物质的溶解度与分离效率与离子液体的氢键碱性密 切相关 ${ }^{[23,25 \sim 31]}$.

溶剂化参数模型方法一定程度上阐明了离子液体 的多重溶剂角色，但作为离子液体这个复杂体系，简 单的实验方法显然不足以窥其全貌. 随着计算技术的 发展, 分子动力学模拟(molecular dynamics simulation) 和量化计算(quantum chemical calculation)方法越来越 多地被用于离子液体体系物化性质的研究. 张锁江 等 ${ }^{[32,33]}$ 通过量化计算等方法, 发现了离子液体中广泛 存在着氢键网络结构(图 1). 结合实验方法，从电子、原 子、分子等多个层次认识了阴阳离子的微观结构与物 化性能的关系，在此基础上揭示了离子液体中的氢键 及其网络扩展性及离子对/团簇聚集结构与物化性能 的关系，从分子水平上揭示了实验观测到的离子超结 构单元的形成机理 ${ }^{[33,34]}$. 氢键网络结构的存在证明了 离子液体并不是简单的完全电离的离子体系. 这些研 究从分子水平上阐明了离子液体不同于传统分子溶剂 或电解质溶液的微观本质, 为发展离子液体的理论模 型提供了科学依据. 为得到离子液体物性的预测方法, Liu 等 ${ }^{[35,36]}$ 、Zhou 等 ${ }^{[37]} 、 Y u$ 等 ${ }^{[38]}$ 、Sun 等 ${ }^{[39]}$ 研究了阴 阳离子的作用位点、氢键、相互作用能和烷基侧链的 转动灵活性等离子液体的液相结构, 建立了离子液体 的微观参数(如氢键、配位数等)与宏观性质(包括密度、

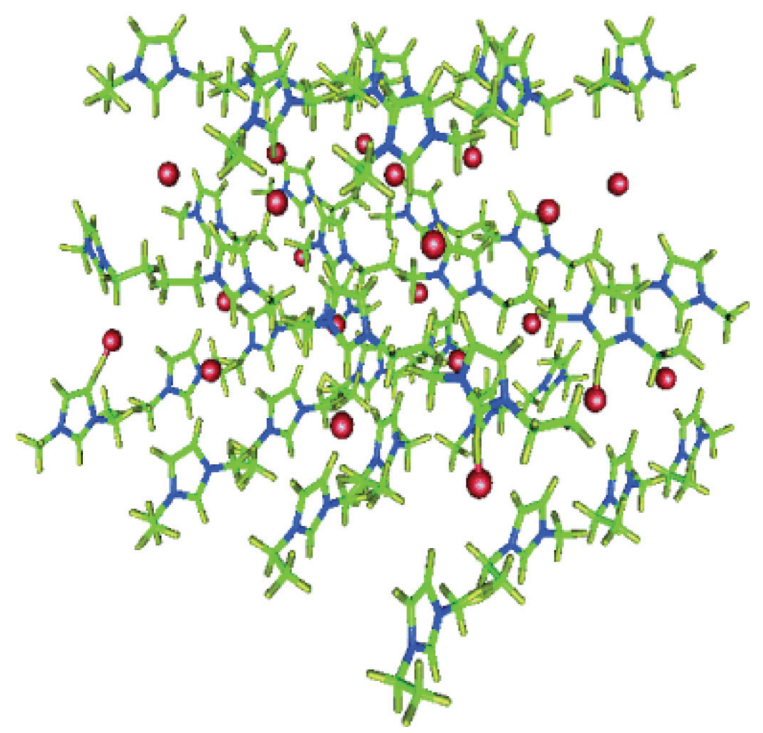

图 1 1-乙基-3-甲基咪唑氯盐([Emim]Cl)离子液体中的氢键 网络示意图 ${ }^{[33]}$ (网络版彩图) 
相变焓、扩散系数等)之间的定量关系, 并结合半经验 的定量结构-性质(QSPR)方法, 形成了对离子液体的 多尺度构效关系的认识. Freire 等 ${ }^{[40]}$ 采用 COSMO-RS 计算方法评估离子液体氢键碱性, 表明离子液体的氢 键碱性和其与溶质分子之间的氢键键能呈正相关. Xing 等 ${ }^{[41,42]}$ 基于量子化学方法, 从电子水平上系统研 究了离子液体结构与碱性的关系. 采用分子表面最小 静电势和最小平均局部离子化能两个量化参数分别描 述了离子液体的静电碱性和共价碱性(图 2), 计算结果 与实验结果相一致. 研究表明, 与中性的分子溶剂相 比, 离子液体独特的静电场赋予其较强的氢键碱性; 而与无机盐相比, 离子液体松散的微观结构使得阴离 子更加“自由”, 从而表现出更强的氢键碱性 ${ }^{[42]}$. 进一 步研究发现, 离子液体阴离子的静电碱性和共价碱性 随着离子液体阴阳离子间相互作用能的降低均明显增 强; 当功能基团键合在阴离子侧时其碱性显著增强 ${ }^{[41]}$. 在此基础上, 提出了两种增强离子液体碱性的方法: 削弱阴阳离子间的相互作用和采用阴离子键合策略.

在离子液体聚集结构方面, Voth 和 Wang 采用多 尺度的粗粒化计算方法(multiscale coarse-graining (MSCG) method)展示了离子液体独特的空间异质性 ${ }^{[43]}$. 其计算结果表明, 带电的阴离子和阳离子侧链头基由 于强静电场的作用而均匀分布, 而电中性的尾基侧则 由于短程作用力而趋于聚集. Rebelo 等 ${ }^{[44]}$ 第一次直接 测定了离子液体的内聚能, 并用分子模拟予以验证, 证明了离子液体阴阳离子之间存在着一定程度的离析, 分别聚集成极性与非极性区域; 非极性域的尺寸随烷

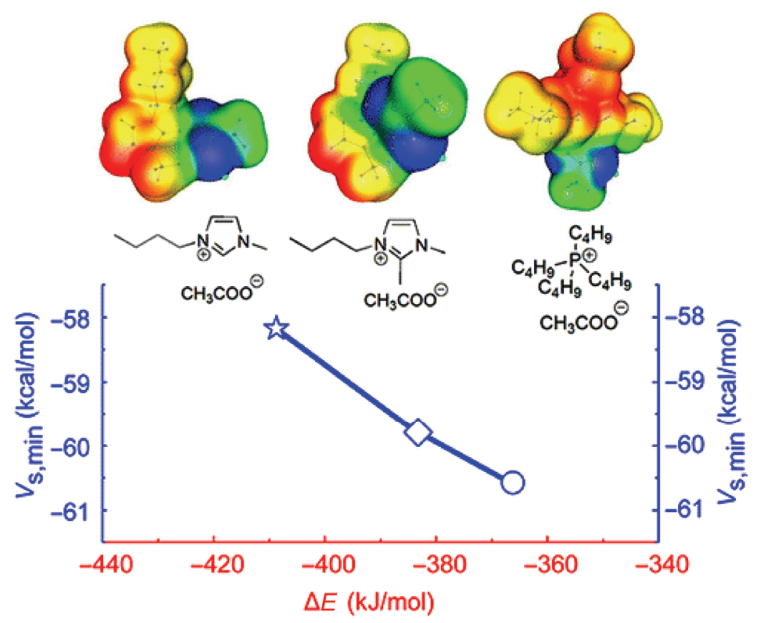

图 2 离子液体的分子表面平均局部离子化能的分布图. 颜 色范围: 红色>黄色>绿色>蓝色 ${ }^{[41]}$ (网络版彩图)
基链的增长而增大, 但不会破坏整个离子网络. 结合 $\mathrm{X}$ 射线散射等手段, 离子液体纳米尺度的微观结构也 进一步被揭示. Triolo 等 ${ }^{[45]}$ 借助于 $X$ 射线衍射技术第 一次实验性地证明了离子液体中存在着纳米尺度的不 均一性. 当咪唑离子液体阳离子侧链上烷基链碳数在 4 10 变动时, 其结构尺度为 14 26 A. 借助于小角和 宽角 $X$ 射线散射, Drummond 等 ${ }^{[46]}$ 调查了 20 种质子型 离子液体的结构性质. 其结果表明大部分离子液体都 具有纳米结构. 通过实验与分子模拟相结合的手段, 研究者揭示出离子液体纳米结构存在的原因很大程度 上归结于阴阳离子之间极性域与非极性域的离析(图 3 ); 当烷基链较长时, 离析程度更为显著 ${ }^{[47 ~ 49]}$. 纳米 结构是离子液体有别于传统分子溶剂的一个重要特性, 也是其得以成为两亲自组装媒介的重要条件 ${ }^{[50]}$. Lodge 等 ${ }^{[51]}$ 研究发现, 1,2-丁二烯嵌段共聚的环氧乙烷 在咪唑离子液体中会发生自聚集而形成有序的纳米胶 束. 研究表明, 溶质分子作用于离子液体时, 极性域 与非极性域离析的离子液体对溶质分子具有特殊的影 响. 极性溶质如水等一般只与极性区域相作用, 而非 极性溶剂如正己烷则反之作用于非极性区域 ${ }^{[52]}$. 相较 于咪唑离子液体弦状的非极性域, 季膦离子液体则更 类似于球状，因此其非极性域对疏水性分子具有更好 的溶解性 ${ }^{[53]}$. 基于此, Yang 等 ${ }^{[54,55]}$ 设计了一类阴离子 功能化的长链脂肪酸纳米结构离子液体, 用于溶解难 溶性药物分子. 具有氢键供体和疏水片段的难溶性药 物分子首先与强碱性的离子液体阴离子通氢键作用形 成复合体, 继而在长烷基链的驱动下与疏水片段二次 组装为更有序的液晶结构, 从而远远超越了一般溶剂 化方法所获得的药物溶解度.
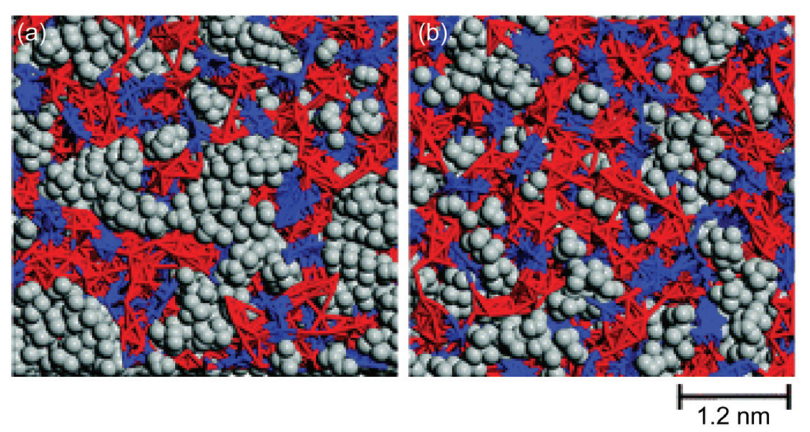

图 3 离子液体极性域(红色)与非极性域(灰色)纳米尺度离 析分子模拟示意图. (a) $\left[\mathrm{C}_{9} \mathrm{mim}\right]\left[\mathrm{NTf}_{2}\right]$ (1-壬基-3-甲基咪唑双 三氟甲烷磺酰亚胺); (b) $\left[\left(\mathrm{C}_{1} \mathrm{OC}_{1}\right)_{3} \mathrm{mim}\right]\left[\mathrm{NTf}_{2}\right]$ (1-三聚乙醚-3甲基咪唑双三氟甲烷磺酰亚胺) ${ }^{[47]}$ (网络版彩图) 


\section{3 天然活性物质的分离}

植物在代谢过程中会产生各类由生物途径合成 的二次代谢产物, 其中不少具有明显的生理活性, 因 而被称为生物活性物质, 具有较高的药用和经济价 值. 但天然产物中的活性成分比较复杂, 存在一系列 结构相近、性质相似的同系物, 不同结构的同系物的 活性往往有差异, 某些单体具有独特的活性, 因此相 似物的分离很有必要. 然而它们的结构差异仅在取 代基的种类和个数、甚至是取代基的位置不同, 因此 溶解度、分子大小等比较接近, 从而导致单体的分离 难度较大. 现有的传统方法如结晶、吸附层析技术等 存在处理量小、溶剂消耗大等不足. 因此, 开发高效 的天然生物活性同系物的分离技术具有重要的意义.

天然维生素 $\mathrm{E}$, 也被称为生育酚, 是生物体内最 重要的脂溶性维生素, 包括 $\alpha-、 \beta-、 \gamma$-和 $\delta$ - 4 种构型, 其中 $\alpha$-生育酚的生理活性显著高于其他几种同系物, 因此将 $\alpha$-生育酚从其他几种生育酚中分离出来很有 必要. 杨启炜等 ${ }^{[56 ~ 58]}$ 选择碱性离子液体作为萃取介 质，借助于离子液体与生育酚的氢键相互作用，通过 识别 4 种同系物微弱的氢键酸性差异, 实现了生育酚 同系物的高选择性分离. 同时发现了离子液体和助 溶剂之间的协同效应, 稀释剂的加入可显著提高离 子液体的萃取能力, 能同时实现高的萃取选择性、合 适的分配系数、较低的黏度和较低的离子液体消耗量. 实验发现, [Bmim]Cl-甲醇混合物为萃取剂时, $\delta$-生育 酚对 $\alpha$-生育酚的萃取选择性系数可高达 21 以上. 乙 腈为稀释剂时与 $[\mathrm{Bmim}] \mathrm{Cl}$ 的复合溶剂为萃取剂, 在 与正已烷组成的两相体系中, $30^{\circ} \mathrm{C}$ 时, $\delta$-生育酚对 $\alpha$ 生育酚的萃取选择性系数达 11.3 , 是纯乙腈作萃取剂 时的 4 倍, $\delta 、 \beta \& \gamma 、 \alpha$-生育酚的分配系数是纯离子液 体为萃取剂时的 18 倍以上. 根据生育酚特殊的酚羟 基基团的特性, $\mathrm{Ni}$ 等 ${ }^{[59]}$ 制备了一系列氨基酸为阴离 子的离子液体, 将离子液体-二甲基甲酰胺(DMF)的 复合溶剂作为萃取剂, 利用液-液萃取将 $\alpha$-生育酚从 与其结构类似的亚油酸甲酯中分离出来， $\alpha$-生育酚对 亚油酸甲酯的选择性系数高达 29.6 , 几乎是以纯 DMF 为萃取剂的 10 倍. 研究发现 ${ }^{[56-59]}$, 在基于离子 液体的复合萃取剂体系中, 存在着“稀释剂的摆动效 应” (dilute-swing effect). 在一定的离子液体浓度时, 存在着萃取分配系数的最大值. 因此, 在萃取分离结 束后，通过简单的减压蒸馏去除分子溶剂或用分子

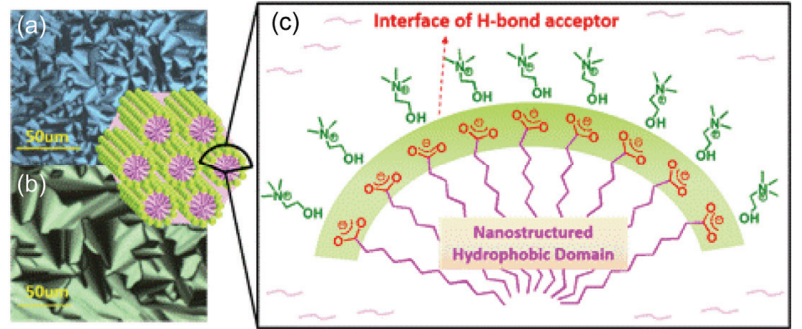

图 4 月桂酸胆碱在 DMSO (a) 和 DMF (b)中形成的溶致液 晶在偏光显微镜 $(\mathrm{POM})$ 中的织构以及相应的微观结构示意 图(c) ${ }^{[60]}$ (网络版彩图)

溶剂进一步稀释可显著降低分离对象在萃取剂中的 分配进而得以高效地反萃回收. 实验证明, 对回收的 离子液体进行多次重复利用，其分离性能基本保持 不变. 依托于离子液体的自组装特性, Liu 等 ${ }^{[60]}$ 设计 利用了具有生物相容性的胆碱脂肪酸离子液体, 其 在助溶剂中可以形成高度有序的液晶结构(图 4), 源 于其特殊的纳米有序结构和强的氢键碱性，显著提 高了生育酚的萃取分配和选择性, 月桂酸胆碱/二甲 基亚砜(DMSO)体系中生育酚对亚油酸甲酯的选择性 系数甚至可达到 250 以上. Wang 等 ${ }^{[61]}$ 合成了以 1 -乙 烯基-3-丁基咪唑溴盐离子液体为基础的聚离子液体, 并引入丙氨酸阴离子, 用于生育酚同系物的分离, 发 现该聚氨基酸型离子液体因其氨基酸阴离子与生育 酚酚羟基的氢键作用远强于卤素阴离子而具有出色 的分离效率; 且该聚离子液体在萃取剂中存在温度 刺激响应, 使得可通过改变温度得到快速回收和再 利用以及萃取相中生育酚的再分离, 避免了溶剂的 大量消耗(图 5).

天然香精油是来源于植物的一类重要活性物质, 其提取的物质往往包含多种萜类物质，且结构和化 学性质极为相似, 传统的有机溶剂难以实现有效的 分离, 且得到的产品往往含有较多其他杂质. Arce 等 ${ }^{[30]}$ 合成了具有较强氢键碱性的[Emim][OMs]（1-乙 基-3-甲基咪唑甲基磺酸盐)离子液体选择性萃取分离 模拟的香精油中的柠檬烯和芳樟醇的混合物. 构建 了柠檬烯-芳樟醇-萃取剂的液液平衡体系, 并与非随 机双液体模型方程关联. 其实验结果发现, 相较于 2丁烯-1,4-丁二醇和 1,2-二乙烯醇等有机溶剂，离子液 体具有最高的选择性, 且得到的产物纯度最高.

Cheong 等 ${ }^{[62]}$ 以含有芳香环的离子液体作为萃 取剂，对多不饱和脂肪酸乙酯有较好的萃取能力. $\mathrm{Li}$ 等 ${ }^{[63,64]}$ 采用固相萃取法富集多不饱和脂肪酸甲酯 


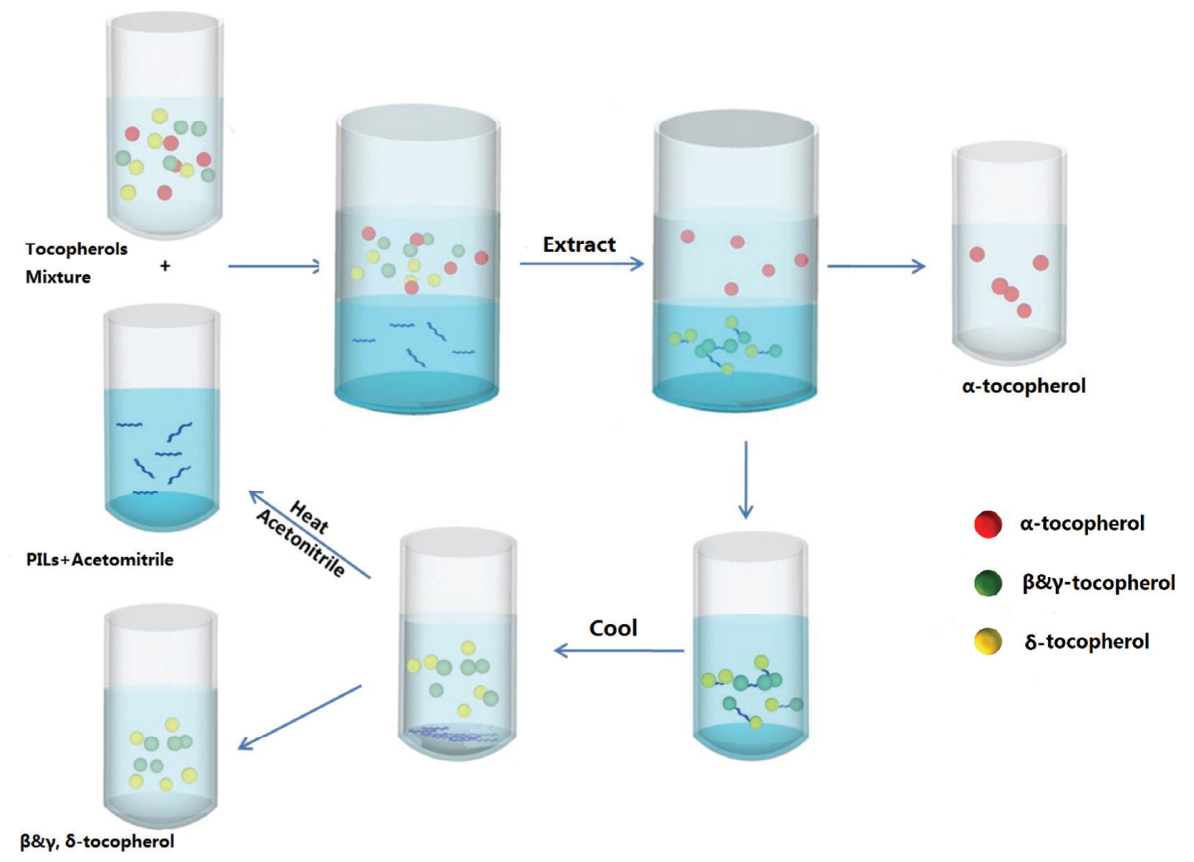

热响应的聚离子液体选择性萃取分离生育酚同系物示意图 ${ }^{[61]}$ (网络版彩图)

(PUFAMEs), 经过实验研究, 二十碳五烯酸(EPA)甲 酯从原料中的 7\%左右增加至 1-已烯洗脱液中的 $90 \%$. 不过此方法虽然可得到纯度较高的 EPA 甲酯产品, 但消耗大量的有机溶剂(如乙醚、己烯等), 使用了昂 贵的银盐, 且制备吸附剂的过程繁琐, 负载于吸附剂 上的离子液体流失较严重, 对于较高的分离选择性, 银发挥了主要作用, 此方法中离子液体本身对双键 的识别作用并不突出. 另外, $\mathrm{Li}$ 等 ${ }^{[65]}$ 以离子液体和银 盐作为萃取介质, 采用液-液萃取的方法对多不饱和 脂肪酸甲酯进行萃取, 向离子液体中加入银盐, 并以 此作为萃取剂. 实验发现加入银盐的离子液体对脂 肪酸甲酯具有较好的分配系数, 这主要是由于 $\mathrm{Ag}^{+}$ 与碳-碳双键发生配合反应, 生成配合物, 有利于脂 肪酸甲酯在萃取相中的分配. 另外, 此研究还发现, 离子液体中阳离子的脂肪链增长, 其分配系数则大 大增加; 阴离子为 $\mathrm{PF}_{6}^{-}$(六氟磷酸) 时多不饱和脂肪酸 甲酯的分配系数大于阴离子 $\mathrm{BF}_{4}{ }^{-}$(四氟硼酸). 此研 究虽然得到了较高的分配系数和分离效果, 但银盐 发挥的作用远远大于离子液体本身, 不能发挥离子 液体的优势, 且银盐比较昂贵. 鉴于银盐和离子液体 易流失的不足, $\mathrm{Li}$ 等 ${ }^{[66,67]}$ 将离子液体固定在吸附剂 SBA-15 或二氧化硅上作为萃取剂, 对多不饱和脂肪
酸甲酯有较好的萃取能力, 并且萃取剂可回收利用.

部分天然活性物质结构上既有芳香环等疏水性 片段、又有羟基等亲水性片段, 分子间内聚能高, 因 此水溶性和油溶性均较差, 呈现疏水疏油的溶解特 性, 使得分离纯化难度更大. Cao 等 ${ }^{[68]}$ 利用离子液体 溶解能力强、易形成液-液两相体系的特点, 成功构建 了离子液体-水-乙酸乙酯新型两相体系, 在离子液体 与稀释剂的协同作用下, 实现了大豆异黄酮、银杏内 酯这两类疏水疏油同系物的高效分离, 为疏水疏油 天然活性同系物的分离制备提供了新的平台. 以 $N$ 乙基吡啶溴盐-水溶液为萃取体系, $30^{\circ} \mathrm{C}$ 时, 大豆苷元 和黄豆黄素对染料木素的分离选择性可达 7.4 和 7.1. 通过量化计算离子液体与银杏内酯的相互作用, 表 现了不同离子液体结构对相互作用的影响, 银杏内 酯与萃取溶剂的氢键作用和范德华作用对银杏内酯 分配系数的影响较明显, 而萃取溶剂的氢键碱性和 偶极/可极化率同时影响银杏内酯同系物的分离选择 性. 实验证明, 在合适的离子液体和浓度下, 银杏内 酯同系物中不仅具有高的选择性, 且萃取容量较高, 原料液处理浓度可达 $25.4 \mathrm{~g} / \mathrm{L}$, 是常规分子溶剂体系 的 10 倍以上 ${ }^{[69]}$. 实验表明, 该体系下同样存在稀释 剂的摆动效应, 萃取完成时, 通过在萃取相中加水稀 
释即可被乙酸乙酯高效反萃, 并通过减压蒸馏回收 离子液体; 回收的离子液体重复使用 5 次, 萃取效率 保持不变. Dong 等 ${ }^{[70]}$ 采用分子模拟和量化计算了离 子液体与 3 种大豆异黄酮苷元与离子液体之间的氢 键相互作用, 发现实验的分配系数与计算得到的最 低势能数据相一致.

部分天然活性物质与其同系物只在不饱和度上 (双键个数) 存在差别, 其分离十分困难, 传统分子溶 剂难以有效识别同系物在不饱和度上的微小差异, 常需要添加金属盐作为分离介质, 存在金属盐易流 失、易失活、成本高等不足. Liang 等 ${ }^{[71,72]}$ 构建了一系 列具有 $\pi-\pi$ 作用能力的离子液体萃取剂, 利用离子液 体对碳碳单键和碳碳双键的选择性识别能力, 在不 添加金属盐的条件下, 实现了胆固醇、辣椒碱这两类 具有不饱和度差异的天然活性同系物的有效分离. 液-液萃取分离实验证明, 离子液体 [Bmim] [SCN] (1丁基-3-甲基咪唑硫氧(酸盐)对胆固醇和 24-去氢胆固 醇两者的选择性达到 2.45 , 而在氢键碱性更强的 [Bmim][OAc] (1-丁基-3-甲基味唑醋酸盐)中, 胆固醇 达到最大的分配系数 5.935 , 这在普通溶剂中难以实 现. 维生素 $\mathrm{D}_{3}$ 和速甾醇 $\mathrm{T}_{3}$ 两组物质在有机溶剂和离 子液体中的萃取行为, 对于维生素 $\mathrm{D}_{3}$ 和速甾醇 $\mathrm{T}_{3}$, 传统有机溶剂中分离效果最好的环丁砜仅能达到 1.44 的选择性系数, 而[Bmpr][NTf $\left.{ }_{2}\right]$ (1-丁基-3-甲基 吡咯双三氟甲烷磺酰亚胺盐)对两者的选择性中可达 到 1.77 .

Row 等 ${ }^{[73]}$ 将离子液体固相微萃技术用于绿茶提 取咖啡因与茶碱. 由于咖啡因与茶碱结构上唯一的 区别是咖啡因化合物的氮原子上存在一个甲基, 分 离上具有一定难度. 他们在聚合物微球上引入 1 -甲 基咪唑离子基团, 削弱了咖啡因与聚离子液体的氢 键作用, 从而能使用聚离子液体对两者进行选择性 分离. 用该方法从绿茶中分离咖啡因和茶碱, 萃取率 分别为 0.025 和 $0.58 \mathrm{mg} / \mathrm{mL}$.

此外, 离子液体也被用于氨基酸 ${ }^{[74,75]}$ 、磷脂 ${ }^{[76]}$ 、 生物碱 ${ }^{[77]}$ 等相似结构的天然活性物质的分离, 取得 了优于常规分子溶剂的分离选择性.

天然活性物质由于分子结构复杂、分子内聚能 高、往往兼具多个官能团, 因此在水和非极性溶剂中 溶解度均十分有限; 且各结构相似物间性质相近, 分 离纯化难度很大. 离子液体因其与天然活性分子之 间的 $\pi-\pi$ 、氢键、范德华和静电等的多重相互作用, 具
有良好的溶解性、极高的分离效率、优异的选择性等 特点, 表现出传统方法不具备的优势. 近年来, 离子 液体在天然活性物质分离领域研究发展迅速, 初步 展示了其在分离过程中的巨大应用潜力. 但是目前 对离子液体的结构参数、毒性、腐蚀性等物理化学性 质的研究数据仍不够完善、对其萃取机制和规律研究 仍显不足; 部分对象仍需添加金属离子以增加选择 性，存在易流失、易失活等不足，一定程度上带来了 成本和环境上的负担.

\section{4 有机化合物的分离}

除了天然活性物质, 自然界和化学工业中还存 在许多结构相似的化合物, 对它们的分离也十分有 必要.

Rogers 等 ${ }^{[78]}$ 构建了 $[\mathrm{Bmim}] \mathrm{Cl} / \mathrm{K}_{3} \mathrm{PO}_{4}$ 的双水相体 系用于分离短链醇类(甲醇、乙醇、丙醇等), 发现其 分配系数随醇链的增长而增大. 进一步研究发现, 在 给定相组成的双水相体系中, 疏水性越强的物质, 其 分配系数越大，因此通过简单调节相组成即可得到 预想的分配效果 ${ }^{[79]}$.

作为世界上最丰富的生物质资源，木质纤维素 是生产清洁能源和精细化学品的天然原料. Wang 等 ${ }^{[80]}$ 在实验室条件下, 系统研究了离子液体与木质 素、纤维素、半纤维素以及它们的模型化合物的相互 作用机制, 根据纤维素、半纤维素以及木质素在各种 离子液体中溶解度的不同, 成功地对生物质原料三 组分进行了分离. 且溶解分离后, 只要加入丙酮等易 与离子液体混合的溶剂, 即可使纤维素沉淀析出, 达 到分离与回收离子液体的目的. 分离纤维素、木质素 以及半纤维素的分离百分比分别为 $99.7 \% 、 75.4 \%$ 、 76.9\%, 溶剂[Bmim][DCA]（1-丁基-3-甲基咪唑双氰 胺盐)和 $[\mathrm{Bmim}][\mathrm{OAc}] / \mathrm{DMSO}$ 的回收率分别为 $89.6 \%$ 和 $99.2 \%$.

$R R R-\alpha$-生育酚聚乙二醇琥珀酸酯(又称 $\mathrm{d}-\alpha$-生育 酚聚乙二醇琥珀酸酯, TPGS)是一种水溶性的维生素 $\mathrm{E}$ 衍生物, 同时具有较强的表面活性容易乳化. 其合 成产物往往是由 TPGS 单酯与双酯组成的混合物, 只 有单酯是其中的有效成分, 因此对单酯与双酯的分 离十分有必要. Kong 等 ${ }^{[81]}$ 在萃取体系中引入离子液 体构建水溶性离子液体与水和有机溶剂的三元两相 体系, 考察了阳离子烷基取代基长度、离子液体浓度 
和阳离子种类的影响, 既有效地解决相对分子量较 大具有表面活性的单双酯混合物分离时的乳化问题, 同时得到的选择性系数大于 10 . 而且利用“稀释剂的 摆动效应”, 萃取结束后用乙酸乙酯反萃就可实现 TPGS 单酯与离子液体的分离和离子液体的重复使用. 离子液体萃取有望成为这一类表面活性高分子化合 物高效分离的通用方法.

芳香烃是指分子结构中含有一个或者多个苯环 的烃类化合物. 石油化工和煤裂解气化过程中, 往往 得到的是芳香烃与脂肪烃的混合物. Matsumoto 等 ${ }^{[82]}$ 测定了苯、甲苯、对二甲苯等在疏水的 $\left[\mathrm{PF}_{6}\right]^{-}$和 $\left[\mathrm{NTf}_{2}\right]^{-}$ 离子液体中的溶解性, 利用离子液体膜材料, 选择性 地分离了庚烷中的 3 种芳香烃. Pereiro 等 ${ }^{[83]}$ 利用具有 芳香性的 1-丁基-1-甲基吡咯烷双三氟甲烷磺酰亚胺 离子液体, 将苯、甲苯、苯乙烯等芳香烃与脂肪族的 正庚烷分离. 由于离子液体与芳香烃存在的 $\pi-\pi$ 相互 作用, 其在分配率和选择性上都远高于传统的环丁 砜、 Larriba 等 ${ }^{[84]}$ 利用同样具有芳香性且黏度较低的 双氰胺和三氰胺([TCA $\left.]^{-}\right)$咪唑离子液体实现了甲苯 与正庚烷的分离. 吴卫泽等 ${ }^{[85]}$ 发现了四丁基磷溴离 子液体与乙酰丙酸形成的低共熔溶剂, 其中乙酰丙 酸中的碳氧双键能够与芳香环发生 $\pi-\pi$ 相互作用, 从 而高效地从正己烷和环己烷中分离甲苯. Arce 等 ${ }^{[86]}$ 利用疏水性的 1-乙基-3-甲基吡咯烷双三氟甲烷磺酰 亚胺离子液体选择性地分离了苯与庚烷. Meindersma 等 ${ }^{[87]}$ 使用 1 - 乙基-3- 甲基咪唑硫酸氢盐 ([Emim] $\mathrm{HSO}_{4}$ )、1-丁基-3-甲基味唑碘盐([Bmim $]_{3}$ )等选择性 分离正庚烷中的甲苯, 以此研究从油田采出水中分 离芳香烃的可能性. 其结果表明, 离子液体要优于传 统的环丁砜, 分离结束后可通过加热的方式将离子 液体中的有机物除去, 从而实现离子液体的回收再 利用. 这些研究结果证明, 离子液体有望取代传统的 挥发性有机溶剂在石油化工中发挥重要作用, 但离 子液体较大的黏度仍是需要考虑的问题.

酚类化合物如苯酚、甲酚和二甲酚等是重要的化 工原料和化工中间体, 主要来自煤加氢液化油和中 低温热解的煤焦油中. 而目前普遍采用的碱水洗脱 法不仅消耗大量的酸碱, 还会产生大量的废水. 大量 的研究 ${ }^{[88}$ 94] 表明, 离子液体能够高效地从煤焦油和 废水中回收酚类化合物. 王键吉等 ${ }^{[12,88,89]}$ 研究了咪唑 类离子液体 1 - 烷基-3-甲基咪唑四氟嗍酸盐 $\left(\left[\mathrm{C}_{n} \operatorname{mim}\right] \mathrm{BF}_{4}, n=4 、 6 、 8\right)$ 对多种酚类和胺类化合物的
萃取性能, 揭示了离子液体与酚和胺类化合物的相 互作用. 其研究表明, 离子液体对酚和胺的萃取分离 是一个自发的、由焓变控制的放热过程. 不过由于酚 差基的酸性较强, 凭借离子液体的氢键碱性识别很 难将苯酚相似物完全分离. 吴卫泽等 ${ }^{[91,92]}$ 研究了咪 唑离子液体用于萃取分离甲苯和已烷等模拟油中的 酚类化合物的性能, 其结果表明, 离子液体阳离子的 烷基链长度和阴离子种类 $\left(\mathrm{Cl}^{-}>\mathrm{Br}^{-}>\mathrm{BF}_{4}^{-}>\mathrm{PF}_{6}^{-}\right)$对分离 性能有较大影响, 电负性较强的卤素阴离子以及短 的烷基取代链可带来萃取效率的提升，其中 [Bmim] Cl 在 $10 \mathrm{~min}$ 内对苯酚的萃取率可达到 $90 \%$. Khachatryan 等 ${ }^{[95]}$ 测定了苯酚、硝基苯酚和二硝基苯 酚在疏水性离子液体 1-丁基-3-甲基咪唑六氟硼酸离 子液体中的分配系数, 发现这些酚类化合物的分配 效果存在显著差异. Deng 等 ${ }^{[96]}$ 将三己基十四烷基氯 化磷离子液体与三氯化铁混合, 制备了具有磁性的 离子液体, 简捷地分离废水中的各种酚类化合物. 且 发现不同酚类分子的分配系数显著不同, 3,5-对氯苯 酚的分配系数高达 6000 以上, 而苯酚则在 100 左右, 为选择性分离酚类化合物提供了可能性. 而且, 酚和 油的沸点较低, 通过简单的减压蒸馏即可分离回收 离子液体，实现了离子液体的高效回收利用.

上述有机化合物的分离, 传统工业中主要使用 层析法、溶剂法和蒸馏法等, 不仅过程冗长、需溶剂 量大, 且对于结构性质相类似成分的分离常受到限 制. 离子液体的高溶解性和分子特异性识别能力, 为 分离这些有机化合物提供了新的研究方向. 不过利 用离子液体的研究尚属起步, 缺乏规律性的系统研 究, 仍存在成本高、黏度大等不足, 但已初步显示了 工业化的应用潜力.

\section{5 低碳烃的分离}

在工业生产的诸多场合还面临着轻烃分离和纯 化问题，如聚合级乙烯和乙炔的制备、天然气的分离 纯化、炼厂干气的分离和回收利用等. 这些低碳烃的 纯度对下游的工艺影响巨大, 而其结构上往往只是 1 个甲基或是双键的差异. 常规介质对低碳烃的分离 选择性较低, 分离过程能耗极大. 因此, 研究对低碳 烃具有较高选择性的新型分离介质具有重要的实际 意义.

目前, 气体在离子液体中的溶解度已有较多的报 
道. 常见的低碳烃包括甲烷、乙烷、乙烯、乙炔、丙 烷、丙烯、丁烷、1-丁烯等. 相关数据列于表 $1^{[97]}$ 中.

Camper 等 ${ }^{[100]}$ 研究了温度为 $40^{\circ} \mathrm{C}$ 时, 乙烷、乙 烯、丙烷、丙烯、丁烷以及丁烯等气体在 5 种咪唑类 离子液体 $[\mathrm{Bmim}]\left[\mathrm{PF}_{6}\right] 、[\mathrm{Bmim}]\left[\mathrm{BF}_{4}\right] 、[\mathrm{Emim}]\left[\mathrm{NTf}_{2}\right]$ 、 [Emim $]\left[\mathrm{CF}_{3} \mathrm{SO}_{3}\right]$ (1-乙基-3-甲基咪唑三氟磺酸盐)和 [Bmim][DCA]中的溶解度. 研究结果表明, 含有不饱 和基团的离子液体通过 $\pi-\pi$ 相互作用可以提高低碳烃 在离子液体中的溶解度, 相同碳数的低碳烃, 在离子 液体中的溶解度随不饱和度的增大而增大. 推测原 因为不饱和烃中的 $\pi$ 电子增强了气体分子和离子液 体间的相互作用. 另外, 由于范德华作用力随气体分 子的碳链增大而增强, 故而碳链较长的气体分子在 离子液体中的溶解度增加. 相关研究 ${ }^{[101,105 ~ 114]}$ 显示, 低碳烃在离子液体中的溶解度大小顺序为 $\mathrm{CH}_{4}<$ $\mathrm{C}_{2} \mathrm{H}_{6}<\mathrm{C}_{2} \mathrm{H}_{4}<\mathrm{C}_{3} \mathrm{H}_{8}<\mathrm{CO}_{2}<\mathrm{C}_{3} \mathrm{H}_{6}<\mathrm{C}_{4} \mathrm{H}_{10}<\mathrm{C}_{4} \mathrm{H}_{8}<\mathrm{C}_{4} \mathrm{H}_{6}<\mathrm{C}_{6} \mathrm{H}_{6}$. Scovazzo 等 ${ }^{[102]}$ 测定了多种气体 $\left(\mathrm{CH}_{4} 、 \mathrm{C}_{2} \mathrm{H}_{6} 、 \mathrm{C}_{2} \mathrm{H}_{4}\right.$ 、 $\mathrm{CO}_{2} 、 \mathrm{C}_{3} \mathrm{H}_{6}$ 等)在 5 种磷酯为阴离子的离子液体中的
溶解、扩散和渗透情况. 研究结果与上述规律符合, 相同碳数的烷烃, 不饱和度越高, 溶解度越大. 同时 也发现, 气体在不同离子液体中的溶解顺序为 $\left[\mathrm{P}_{(14) 444}\right][\mathrm{DBS}]$ (三丁基十四烷基十二烷基膦苯磺酸盐) $<\left[\mathrm{P}_{2444}\right][\mathrm{DEP}]$ (三丁基乙基膦磷酸二乙酯盐) $<$ $\left[\mathrm{P}_{(14) 666]}\right][\mathrm{DCA}]$ (三己基十四烷基双氧胺盐)< $\left[\mathrm{P}_{(14) 666}\right][\mathrm{Cl}]$ (三己基十四烷基氯盐) $<\left[\mathrm{P}_{(14) 666}\right]\left[\mathrm{NTf}_{2}\right]$ (三己基十四烷基双三氟甲烷磺酰亚胺盐). 同时, 在 对 $\left[\mathrm{NTf}_{2}\right]$ 型季铵型离子液体的比较中发现, 随着其阳 离子的烷基链上的碳数增加, 气体的溶解度也随之 增加. 推测导致该现象的原因是烷基链的增长使得 离子液体的自由体积变大, 有利于气体的溶解, 即空 穴理论 ${ }^{[101]}$. 此外, Zhang 等 ${ }^{[115]}$ 研究发现, 用甲基取代 咪唑环 $\mathrm{C} 2$ 位上的酸性的 $\mathrm{H}$ 原子后, 乙烯的溶解度增 加; 而在咪唑环的 $\mathrm{C} 1$ 位引入氧丙基则会使乙烯的溶 解度下降.

离子液体在乙炔和乙烯分离方面显示了良好的

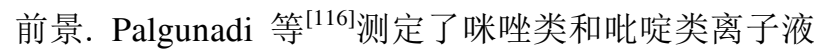

表 1 低碳烃在不同离子液体中的亨利系数(bar $)^{[97]}$

\begin{tabular}{|c|c|c|c|c|c|c|c|c|c|}
\hline ILs & 温度 $\left({ }^{\circ} \mathrm{C}\right)$ & $\mathrm{CH}_{4}$ & $\mathrm{C}_{2} \mathrm{H}_{4}$ & $\mathrm{C}_{2} \mathrm{H}_{6}$ & $\mathrm{C}_{3} \mathrm{H}_{6}$ & $\mathrm{C}_{3} \mathrm{H}_{8}$ & 1- $\mathrm{C}_{4} \mathrm{H}_{8}$ & $\mathrm{C}_{4} \mathrm{H}_{10}$ & Ref. \\
\hline$\left[\mathrm{P}_{4444}\right]\left[\mathrm{C}_{5} \mathrm{H}_{11} \mathrm{COO}\right]$ & 35 & 223.9 & & 45.0 & & 16.0 & & & \\
\hline$\left[\mathrm{P}_{4442}\right]\left[\mathrm{C}_{5} \mathrm{H}_{11} \mathrm{COO}\right]$ & 35 & 239.8 & & 44.1 & & 19.0 & & & \\
\hline$\left[\mathrm{P}_{4442}\right]\left[\mathrm{C}_{11} \mathrm{H}_{23} \mathrm{COO}\right]$ & 35 & 171.3 & & 28.3 & & 13.2 & & & \\
\hline$\left[\mathrm{P}_{4442}\right]\left[\mathrm{C}_{17} \mathrm{H}_{35} \mathrm{COO}\right]$ & 35 & 140.2 & & 25.2 & & 10.5 & & & \\
\hline$\left[\mathrm{P}_{4444}\right]\left[\mathrm{C}_{17} \mathrm{H}_{35} \mathrm{COO}\right]$ & 35 & 145.8 & & 26.1 & & 10.9 & & & \\
\hline$\left[\mathrm{P}_{(14) 666}\right][\mathrm{TMPP}]$ & 40 & 78.0 & & 19.0 & & 6.4 & & & [98] \\
\hline$\left[\mathrm{P}_{4444}\right][\mathrm{TMPP}]$ & 40 & 124.0 & & 29.0 & & 10.0 & & & [98] \\
\hline$\left[\mathrm{P}_{8111}\right][\mathrm{TMPP}]$ & 40 & 130.8 & & 28.2 & & 9.7 & & & [98] \\
\hline$\left[\mathrm{P}_{4444}\right]\left[\mathrm{C}_{5} \mathrm{H}_{11} \mathrm{COO}\right]$ & 40 & 224.7 & 44.9 & 49.5 & & 17.5 & & & [99] \\
\hline$[$ Emim] $]\left[\mathrm{NTf}_{2}\right]$ & 40 & 546.0 & 122 & 169.0 & & 85.0 & & & {$[100]$} \\
\hline$[\mathrm{Bmim}]\left[\mathrm{PF}_{6}\right]$ & 40 & & 188.0 & 341.0 & 74.1 & 187.0 & 40.4 & 85.6 & {$[100]$} \\
\hline$[\mathrm{Bmim}]\left[\mathrm{BF}_{4}\right]$ & 40 & & 253.0 & 415.5 & 88.0 & 251.0 & 55.0 & 111.0 & {$[100]$} \\
\hline$[\mathrm{Emim}]\left[\mathrm{CF}_{3} \mathrm{SO}_{3}\right]$ & 40 & & 213.5 & 355.0 & 87.8 & 209.0 & 47.8 & 96.4 & {$[100]$} \\
\hline$[$ Emim $][\mathrm{DCA}]$ & 40 & & 337.0 & 656.5 & 124.5 & 304.5 & 72.0 & 170.5 & {$[100]$} \\
\hline$\left[\mathrm{P}_{(14) 666}\right][\mathrm{Cl}]$ & 30 & & 35.0 & & 12.0 & & 4.8 & & {$[101]$} \\
\hline$\left[\mathrm{P}_{(14) 666}\right]\left[\mathrm{NTf}_{2}\right]$ & 30 & & 25.0 & & 8.9 & & 4.3 & & [101] \\
\hline$\left[\mathrm{P}_{(14) 666}\right][\mathrm{DCA}]$ & 30 & & 36.0 & & 11.5 & & 6.0 & & {$[101]$} \\
\hline$\left[\mathrm{N}_{(4) 111}\right]\left[\mathrm{NTf}_{2}\right]$ & 30 & & 108.0 & & 46.0 & 46 & 31.4 & & {$[102]$} \\
\hline$\left[\mathrm{N}_{(6) 111}\right]\left[\mathrm{NTf}_{2}\right]$ & 30 & & 99.0 & & 27.0 & 22 & 28.4 & & {$[102]$} \\
\hline$\left[\mathrm{N}_{(10) 111}\right]\left[\mathrm{NTf}_{2}\right]$ & 30 & & 73.0 & & 28.0 & 15 & 16.2 & & {$[102]$} \\
\hline$\left[\mathrm{N}_{(4) 113}\right]\left[\mathrm{NTf}_{2}\right]$ & 30 & & 104 & & 44 & 30 & 22.3 & 12 & {$[102]$} \\
\hline$\left[\mathrm{N}_{(6) 113}\right]\left[\mathrm{NTf}_{2}\right]$ & 30 & & & & 33 & 25 & 21.3 & 12 & {$[102]$} \\
\hline$\left[\mathrm{N}_{(10) 113}\right]\left[\mathrm{NTf}_{2}\right]$ & 30 & & 70 & & 25 & & & 7.8 & {$[102]$} \\
\hline$\left[\mathrm{N}_{(1) 888}\left[\mathrm{NTf}_{2}\right]\right.$ & 30 & & 37.0 & & 12.0 & 7.2 & 6.9 & & {$[102]$} \\
\hline$\left[\mathrm{N}_{(6) 222}\right]\left[\mathrm{NTf}_{2}\right]$ & 30 & & 65.0 & & 31.0 & 20.0 & 17.2 & & {$[102]$} \\
\hline$[\mathrm{Bmim}]\left[\mathrm{MeSO}_{4}\right]$ & 20 & 345.0 & & & & & & & [103] \\
\hline$[\mathrm{Hmim}]\left[\mathrm{NTf}_{2}\right]$ & 20 & 183.0 & & & & & & & {$[104]$} \\
\hline
\end{tabular}


体用于吸收单组分的乙炔和乙烯的性能. 研究发现, 在所用的 13 种离子液体中, 乙炔的溶解度均高于乙 烯. 并且阴离子的碱性越强, 对乙炔的吸收效果越好. 此外研究表明, 摩尔体积较大的离子液体对乙烯的 吸收效果相对更好, 如 [Emim] $\left[\mathrm{NTf}_{2}\right]$. 通过量子化学 计算的方法, Cheong 等 ${ }^{[117,118]}$ 简单构建了乙炔与咪唑 离子液体之间的相互作用构型, 发现乙炔分子与离 子液体阳离子几乎没有作用, 而与阴离子则有一定 的亲和作用. 邢华斌等 ${ }^{[119,120]}$ 采用量化计算和分子动 力学模拟研究了乙烯和乙炔在咪唑离子液体中的溶 解行为, 从分子层次上揭示了乙炔和乙烯在离子液 体中溶解机理的差异. 结果表明, 离子液体与乙炔之 间的氢键作用是决定乙炔溶解度的首要因素, 但乙 烯在离子液体中溶解度受到氢键作用、 $\mathrm{p}-\pi$ 作用和 $\pi-\pi$ 作用的共同影响(图 6). 计算结果与实验结果规律相 一致. 该课题组 ${ }^{[99,121]}$ 进一步借助 COSMO-RS 方法对 离子液体进行高通量的设计和篮选(图 7), 设计具有 强碱性、高自由体积的季膦型长链脂肪酸离子液体, 实现了乙炔乙烯的高容量高选择性的分离. $298.1 \mathrm{~K}$

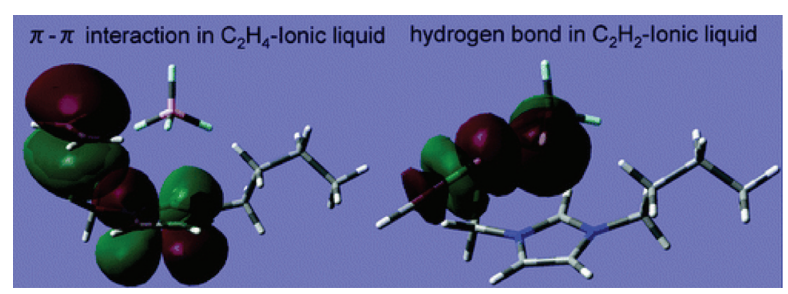

图 6 乙炔与离子液体的氢键作用和乙烯与离子液体的 $\pi-\pi$ 作用 ${ }^{[119]}$ (网络版彩图)

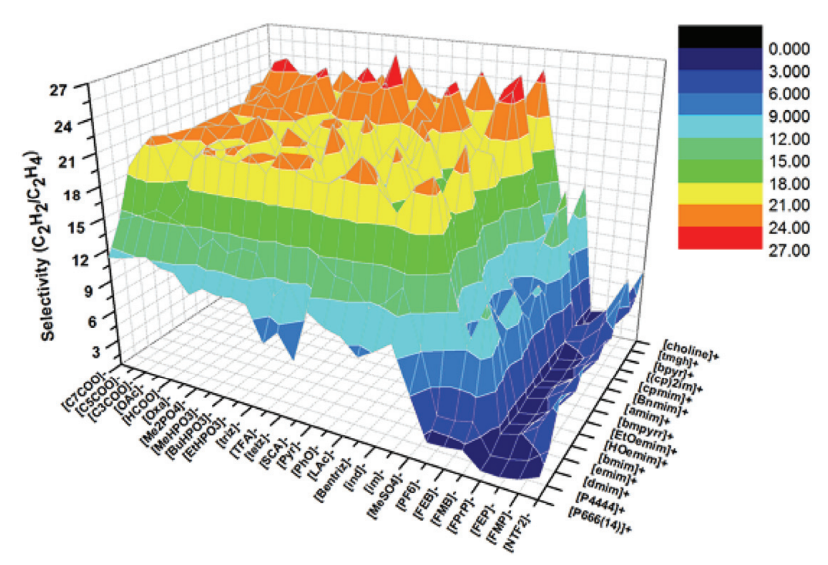

图 7 COSMO-RS 计算预测 $313.15 \mathrm{~K}$ 下 420 种离子液体的 乙炔/乙烯选择性 ${ }^{[99]}$ (网络版彩图)
时, 乙炔在四丁基膦正己酸盐 $\left(\left[\mathrm{P}_{4444}\right]\left[\mathrm{C}_{5} \mathrm{COO}\right]\right)$ 中的 摩尔吸收容量可达到 $0.476 \mathrm{bar}^{-1}$, 是目前工业上常用 吸收溶剂 $N$-甲基吡咯烷酮的 4 倍, 且乙炔和乙烯的选 择性达到 21.4, 而且离子液体热稳定性高, 可以同时 采用减压与加热的方法解吸气体，更为简便与彻底 地回收离子液体, 多次吸收-解吸循环使用吸收容量 基本保持不变.

相较而言, 低碳饱和烷烃在离子液体中的溶解 度较低 ${ }^{[98,102,114,115,122 \sim 124]}$. $293.3 \mathrm{~K}$ 时, $\mathrm{CH}_{4} 、 \mathrm{C}_{2} \mathrm{H}_{6}$ 和 $\mathrm{C}_{3} \mathrm{H}_{8}$ 在 $[\mathrm{Bmim}]\left[\mathrm{PF}_{6}\right]$ 中的亨利系数分别仅为 $693 、 221$ 和 $190 \mathrm{bar} ; 313.1 \mathrm{~K}$ 时, $\mathrm{CH}_{4}$ 和 $\mathrm{C}_{2} \mathrm{H}_{6}$ 在 $[\mathrm{Bmim}]\left[\mathrm{BF}_{4}\right]$ 中 的亨利系数分别仅为 1110 和 354.2 bar. 对于工业应 用而言, 上述的吸收情况并不理想. 近年来, Liu 等 ${ }^{[98,125,126]}$ 设计合成了一类以烷基磷酸为阴离子的离 子液体, 显著提高了低碳烷烃的溶解度, 吸收容量明 显优于阴离子为 $\mathrm{BF}_{4}^{-} 、 \mathrm{PF}_{6}^{-} 、 \mathrm{DCA}^{-}$和 $\mathrm{NTf}_{2}{ }^{-}$等的离子 液体; 而对于相同的烷基磷酸阴离子, 低碳烷烃的溶 解度则随着阳离子上碳原子数目的增加而增加. 313 $\mathrm{K}$ 时, $\mathrm{CH}_{4} 、 \mathrm{C}_{2} \mathrm{H}_{6}$ 和 $\mathrm{C}_{3} \mathrm{H}_{8}$ 在 $\left[\mathrm{P}_{(14) 666}\right]$ [TMPP] (三己基十 四烷基膦双 2,4,4-三甲基戊基磷酸盐)中的亨利系数 分别达到 78、19 和 6.4 bar. 但该类离子液体的黏度 较大. 针对这一问题, 本课题组合成了黏度较低的 $\left[\mathrm{P}_{4442}\right]$ (三丁基乙基膦)型羧酸离子液体, 在对饱和烷 烃的溶解度优于传统离子液体的同时, 保持较低的 黏度. Liu 等 ${ }^{[127]}$ 采取双离子液体的办法, 将具有大吸 收容量的 $\left[\mathrm{P}_{(14) 666}\right][\mathrm{TMPP}]$ 和一种低黏度的质子型离子 液体 $[\mathrm{BHim}][\mathrm{OAc}]$ (1-丁基咪唑醋酸盐)按照不同的比 例混合. 实验结果表明, 该策略在有效降低黏度的同 时, 依然可以维持较低的亨利系数, 但是质子型的 $\left[\right.$ BHim] $[\mathrm{OAc}]$ 不稳定, 容易挥发. 此外, Hert 等 ${ }^{[128]}$ 测 定了 $\mathrm{CH}_{4}$ 在 $[\mathrm{Hmim}]\left[\mathrm{NTf}_{2}\right]$ 中的溶解度, 并比较了 $\mathrm{CO}_{2}$ 对其溶解的影响, 结果发现, $\mathrm{CO}_{2}$ 能增加 $\mathrm{CH}_{4}$ 在该离 子液体中的溶解度.

现有的低碳烃在离子液体溶解度和分离性能的 初步研究展现了离子液体可应用于低碳烃分离过程 的潜力. 特别是功能化的离子液体具有较强的氢键 碱性以及 $\pi$ 电子效应等, 可精细调控饱和及不饱和烷 烃的吸收分离, 显现了优于传统有机溶剂的分离能 力. 但离子液体的高黏度不利于气液两相间的混合 传质, 一定程度上限制了其应用, 其经济性有待进一 步评估. 


\section{6 结论与展望}

室温离子液体是一类“可设计”的溶剂，基于离 子液体与溶质分子的多重相互作用, 引入特定结构 的功能化基团, 精细调控其结构和性质, 可实现对特 定化合物的选择性分离. 一方面, 离子液体可与溶质 发生疏水、氢键、 $\pi-\pi$ 、静电等相互作用, 具有溶解度 大和选择性好等优点; 另一方面, 离子液体的极低的 挥发性和较强的离子内聚能也较大程度上减少了分 离过程气-液、液-液两相间的交叉污染, 便于分离介 质的循环利用, 发展绿色分离方法. 现有的研究初步 展现了离子液体应用于结构相似物分离过程的潜力. 但对于真正的工业应用而言, 离子液体结构相似物 分离过程仍存在一些亟需解决和完善的问题. 未来 的研究方向包括:

(1) 系统地了解和掌握离子液体与目标化合物 间相互作用机理, 利用离子液体独特的纳米聚集结 构和自组装性质, 构建具有有序纳米结构的功能性 软材料, 提高对结构相似物的分子识别能力, 掌握离 子液体结构对分离效率和选择性的影响规律, 指导 高效功能化离子液体的设计和定制, 设计出新型离 子液体及离子液体为介质的分离体系.
（2）离子液体的黏度普遍较高, 不利于气-液和 液-液两相间的扩散传质。高黏度体系的过程强化方 法, 应当引起重视. 同时, 研究离子液体的构效关系 以及离子液体体系的扩散传递规律, 合成低黏度的 离子液体或离子溶液, 发展离子液体杂化材料探索 分离新模式是今后值得发展的方向 ${ }^{[129]}$.

(3) 目前而言, 离子液体的成本相对较高, 与现 有成熟的常规有机溶剂相比, 其经济可行性有待于 进一步评价，这也是制约工业应用的一大瓶颈. 为了 降低其在分离过程的使用成本，一是需要推动离子 液体的规模化生产, 从整体上降低离子液体的生产 成本; 二是要研发清洁高效的离子液体循环回收的 方法; 三要积极探索离子液体在高附加值产品分离 纯化过程中的应用，提高过程的技术经济可行性. 此 外, 在离子液体的应用过程中, 需系统评价离子液体 对生态和人体的安全性, 发展兼具高分离效率和低 环境影响的新型离子液体。

20 多年来, 围绕离子液体的基础研究已经积累 了大量的成果，为工业化应用奠定了良好的基础. 离子 液体的“可设计性”, 反映了它的“普适性”，这正是分 离工程领域最需要具备的特点. 可以期待, 离子液体 为介质的分离技术必将取代更多的传统的分离工艺.

\section{参考文献}

1 Earle MJ, Esperanga JMSS, Gilea MA, Lopes JNC, Rebelo LP, Magee JW, Seddon KR, Widegren JA. Nature, 2006, 439: 831-834

2 Rogers RD, Seddon KR. Science, 2003, 302: 792-793

3 Blanchard LA, Hancu D, Beckman EJ, Brennecke JF. Nature, 1999, 399: 28-29

4 Earle MJ, Seddon KR. Pure Appl Chem, 2000, 72: 1391-1398

5 Plechkova NV, Seddon KR. Chem Soc Rev, 2008, 37: 123-150

6 Smiglak M, Metlen A, Rogers RD. Acc Chem Res, 2007, 40: 1182-1192

Hallett JP, Welton T. Chem Rev, 2011, 111: 3508-3576

8 Armand M, Endres F, MacFarlane DR, Ohno H, Scrosati B. Nat Mater, 2009, 8: 621-629

Davis JH Jr. Chem Lett, 2004, 33: 1072-1077

10 Huddleston JG, Willauer HD, Swatloski RP, Visser AE, Rogers RD. Chem Commun, 1998, 16: 1765-1766

11 Poole CF, Poole SK. J Chromatogr A, 2010, 1217: 2268-2286

12 Fan J, Fan YC, Pei YC, Wu K, Wang JJ, Fan MH. Sep Purif Technol, 2008, 61: 324-331

13 Hoogerstraete TV, Onghena B, Binnemans K. J Phys Chem Lett, 2013, 4: 1659-1663

14 Hoogerstraete TV, Wellens S, Verachtert K, Binnemans K. Green Chem, 2013, 15: 919-927

15 Li HX, Li Z, Yin JM, Li CP, Chi YS, Liu QS, Zhang XL, Urs WB. Green Chem, 2012, 14: 1721-1727

16 Tang BK, Bi WT, Tian ML, Row KH. J Chromatogr B, 2012, 904: 1-21

17 Bakonyi P, Nemestóthy N, Bélafi-Bakó KB. Int J Hydrogen Energ, 2013, 38: 9673-9687

18 Li P, Paul DR, Chung TS. Green Chem, 2012, 14: 1052-1063

19 Rebelo LPN, Lopes JNC, Esperança J MSS, Guedes HJR, Lachwa J, Najdanovic-Visak V, Visak ZP. Acc Chem Res, 2007, 40: 1114-1121 
Aki SNVK, Brennecke JF, Samanta A. Chem Commun, 2001, 413-414

Anderson JL, Ding J, Welton T, Armstrong DW. J Am Chem Soc, 2002, 124: 14247-14254

Crowhurst L, Mawdsley PR, Perez-Arlandis JM, Salter PA, Welton T. Phys Chem Chem Phys, 2003, 5: 2790-2794

Guo Z, Lue BM, Thomasen K, Meyer AS, Xu XB. Green Chem, 2007, 9: 1362-1373

Swatloski RP, Spear SK, Holbrey JD, Rogers RD. J Am Chem Soc, 2002, 124: 4974-4975

Xie HB, Li SH, Zhang SB. Green Chem, 2005, 7: 606-608

Brandt A, Hallett JP, Leak DJ, Murphy RJ, Welton T. Green Chem, 2010, 12: 672-679

Wang CM, Cui GK, Luo XY, Xu YJ, Li HR, Dai S. J Am Chem Soc, 2011, 133: 11916-11919

Wang CM, Luo XY, Luo HM, Jiang DE, Li HR, Dai S. Angew Chem Int Ed, 2011, 50: 4918-4922

Zhang SJ, Sun J, Zhang XC, Xin JY, Miao QQ, Wang JJ. Chem Soc Rev, 2014, 43: 7838-7869

Arce A, Marchiaro A, Rodriguez O, Soto A. AIChE J, 2006, 52: 2089-2097

Kim YH, Choi YK, Park J, Lee S, Yang YH, Kim HJ, Park TJ, Kim YH, Lee SH. Bioresour Technol, 2012, 109: 312-315

Dong K, Zhang SJ, Wang DX, Yao XQ. J Phys Chem A, 2006, 110: 9775-9782

Dong K, Zhang SJ. Chem Eur J, 2012, 18: 2748-2761

Dong K, Song YT, Liu XM, Cheng WG, Yao XQ, Zhang SJ. J Phys Chem B, 2012, 116: 1007-1017

Liu XM, Zhang SJ, Zhou GH, Wu GW, Yuan XL, Yao XQ. J Phys Chem B, 2006, 11: 12062-12071

Liu XM, Zhou GH, Zhang SJ, Wu GW, Yu GR. J Phys Chem B, 2007, 11: 5658-5668

Zhou GW, Liu XM, Zhang SJ, Yu GR, He HY. J Phys Chem B, 2007, 111: 7078-7084

Yu GR, Zhang SJ, Yao XQ, Zhang JM, Dong K, Dai W, Mori R. Ind Eng Chem Res, 2006, 45: 2875-2880

Sun N, He XH, Dong K, Zhang XP, Lu XM, He HY, Zhang SJ. Fluid Phase Equilib, 2006, 246: 137-142

Cláudio AFM, Swift L, Hallett JP, Welton T, Coutinho JAP, Freire MG. Phys Chem Chem Phys, 2014, 16: 6593-6601

Xu D, Yang QW, Su BG, Bao ZB, Ren QL, Xing HB. J Phys Chem B, 2014, 118: 1071-1079

Yang QW, Xing HB, Bao ZB, Su BG, Zhang ZG, Yang YW, Dai S, Ren QL. J Phys Chem B, 2014, 118: 3682-3688

Wang YT, Voth GA. J Am Chem Soc, 2005, 127: 12192-12193

Santos LMNBF, Lopes JNC, Coutinho JAP, Esperança JMSS, Gomes LR, Marrucho IM, Rebelo LPN. J Am Chem Soc, 2007, 129: 284-285

Triolo A, Russina O, Bleif HJ, Cola ED. J Phys Chem B, 2007, 111: 4641-4644

Greaves TL, Kennedy DF, Mudie ST, Drummond CJ. J Phys Chem B, 2010, 114: 10022-10031

Shimizu K, Bernardes CES, Triolo Alessandro, Lopes JNC. Phys Chem Chem Phys, 2013, 15: 16256-16262

Russina O,Triolo A, Gontrani L, Caminiti R. J Phys Chem Lett, 2012, 3: 27-33

Triolo A, Russina O, Caminiti R, Shirota H, Lee HY, Santos CS, Murthy NS, Castner EW Jr. Chem Commun, 2012, 48: 4959-4961

Greaves TL, Drummond CJ. Chem Soc Rev, 2013, 42: 1096-1120

He YY, Li ZB, Simone P, Lodge TP. J Am Chem Soc, 2006, 128: 2745-2750

Lopes JNC, Padua AAH. J Phys Chem B, 2006, 110: 3330-3335

Blesic M, Lopes JNC, Gomes MFC, Rebelo LPN. Phys Chem Chem Phys, 2010, 12: 9685-9692

Yang QW, Xu D, Zhang JZ, Zhu YM, Zhang ZG, Qian C, Ren QL, Xing HB. ACS Sustain Chem Eng, 2015, 3: 309-316

Jin WB, Yang QW, Bao ZB, Zhang ZG, Ren QL, Yang YW, Xing HB. Chem Commun, 2015, 51: 13170-13173

Yang QW, Xing HB, Su BG, Bao ZB, Wang J, Yang YW, Ren QL. AIChE J, 2013, 59: 1657-1667

Yang QW Xing HB, Cao YF, Su BG, Yang YW, Ren QL. Ind Eng Chem Res, 2009, 48: 6417-6422

Yang QW, Xing HB, Su BG, Yu K, Bao ZB, Yang YW, Ren QL. Chem Eng J, 2012, 181: 334-342

Ni XL, Xing HB, Yang QW, Wang J, Su BG, Bao ZB, Yang YW, Ren QL. Ind Eng Chem Res, 2012, 51: 6480-6488

Liu XX, Yang QW, Bao ZB, Su BG, Zhang ZG, Ren QL, Yang YW, Xing HB. Chem Eur J, 2015: 21: 9150-9156

Lu YY, Yu G, Wang WJ, Ren QL, Li BG, Zhu SP. Macromolecules, 2015, 48: 915-924

Cheong LZ, Guo Z, Yang ZY, Chua SC, Xu XB. J Agric Food Chem, 2011, 59: 8961-8967

Li M, Li TY. Sep Sci Technol, 2008, 43: 2072-2089

Li M, Pham PJ, Wang T, Pittman CU, Li TY. Sep Purif Technol, 2009, 66: 1-8

Li M, Pittman CU, Li TY. Talanta, 2009, 78: 1364-1370

Li M, Pham PJ, Pittman CU, Li TY. Microporous Mesoporous Mater, 2009, 117: 436-443

Li M, Pham PJ, Wang T, Pittman CU, Li TY. Bioresour Technol, 2009, 100: 6385-6390

Cao YF, Xing HB, Yang QW, Bao ZB, Su BG, Yang YW, Ren QL. J Agr Food Chem, 2012, 60: 3432-3440 
Cao YF, Xing HB, Yang QW, Su BG, Bao ZB, Zhang RH, Yang YW, Ren QL. Green Chem, 2012, 14: 2617-2625

Dong K, Cao YF, Yang QW, Zhang SJ, Xing HB, Ren QL. Ind Eng Chem Res, 2012, 51: 5299-5308

梁瑞斯. 以离子液体为介质萃取分离甾醇类物质的研究. 硕士学位论文. 杭州: 浙江大学, 2013.43-60

Liang RS, Bao ZB, Su BG, Xing HB, Yang QW, Yang YW, Ren QL. J Agr Food Chem, 2013, 61: 3479-3487

Tian ML, Yan H, Row KH. Anal Lett, 2010, 43: 110-118

Smirnova SV, Torocheshnikova II, Formanovsky AA, Pletnev IV. Anal Bioanal Chem, 2004, 378: 1369-1375

Wang JJ, Pei YC, Zhao Y, Hu ZG. Green Chem, 2005, 7: 196-202

Zhang JZ, Yu K, Yang QW, Bao ZB, Zhang ZG, Yang YW, Ren QL, Xing HB. RSC Adv, 2015, 5: 77581-77588

曹义风. 离子液体萃取分离疏水疏油天然活性同系物. 博士学位论文. 杭州: 浙江大学, 2013. 109-124

Gutowski KE, Broker GA, Willauer HD, Huddleston JG. Swatloski RP, Holbrey JD, Rogers RD. J Am Chem Soc, 2003, 125: 6632-6633

Bridges NJ, Gutowski KE, Rogers RD. Green Chem, 2007, 9, 177-183

郑勇, 轩小朋, 许爱荣, 郭蒙, 王键吉. 化学进展, 2009, 21: 1807-1812

Kong LY, Yang QW, Xing HB, Su BG, Bao ZB, Zhang ZG, Yang YW, Ren QL. Green Chem, 2014, 16: 102-107

Matsumoto M, Inomoto Y, Kondo K. J Membrane Sci, 2005, 246: 77-81

Pereiro AB, Rodríguez A. AIChE J, 2010, 56: 381-386

Larriba M, Navarro P, García J, Rodríguez F. Ind Eng Chem Res, 2013, 52 : 2714-2720

Hou YC, Li ZY, Ren SH, Wu WZ. Fuel Process Technol, 2015, 135: 99-104

Arce A, Earle MJ, Rodríguez H, Rodríguez H, Seddon KR. Green Chem, 2007, 9: 70-74

Meindersma GW, Podt A, Haan AB. Fuel Processing Technol, 2005, 87: 59-79

裴渊超, 武坤, 王键吉. 河南师范大学学报: 自然科学版, 2006, 34: 219

Pei YC, Wang JJ, Wu K, Zhao Y, Fan J. Z Phys Chem, 2007, 221: 825-835

李闲, 张锁江, 张建敏, 陈玉焕, 张延强, 孙宁. 过程工程学报, 2005, 5: 148-151

侯玉翠, 彭威, 杨春梅, 李省云, 吴卫泽. 化工学报, 2013, 64: 118-123

Hou YC, Ren YH, Peng W, Ren SH, Wu WZ. Ind Eng Chem Res, 2013, 52: 18071-18075

Balasubramanian A, Venkatesan S. Desalination, 2012, 289: 27-34

Nosrati S, Jayakumar NS, Hashim MA. J Hazard Mater, 2011, 192: 1283-1290

Khachatryan KS, Smirnova S, Torocheshnikova II, Shvedene NV, Formanovsky AA, Pletnev IV. Anal Bioanal Chem, 2005, 38: 464-470

Deng N, Li M, Zhao LJ, Lu CF, Rooy SL, Warner IM. J Hazard Mater, 2011, 192: 1350-1357

赵旭，邢华斌，李如龙，杨启炜，苏宝根，任其龙. 化学进展, 2011, 23: 2258-2268

Liu XY, Prausnitz JM. Ind Eng Chem Res, 2013, 52: 14975-14978

Zhao X, Yang QW, Xu D, Bao ZB, Zhang Y, Su BG, Ren QL, Xing HB. AIChE J, 2015, 6: 2016-2027

Camper D, Becker C, Koval C, Noble R. Ind Eng Chem Res, 2005, 44: 1928-1933

Ferguson L, Scovazzo P. Ind Eng Chem Res, 2007, 46: 1369-1374

Condenarin R, Scovazzo P. Chem Eng J, 2009, 147: 51-57

Kumełan J, Kamps AP, Tuma D, Maurer G. J Chem Eng Data, 2007, 52: 2319-2324

Kumełan J, Kamps AP, Tuma D, Maurer G. Ind Eng Chem Res, 2007, 46: 8236-8240

Anthony JL, Anderson JL, Maginn EJ, Brennecke JF. J Phys Chem B, 2005, 109: 6366-6374

Jacquemin J, Husson P, Majer V, Gomes MFC. Fluid Phase Equilib, 2006, 240: 87-95

Hong G, Jacquemin J, Deetlefs M, Hardacre C, Husson P, Gomes GMC. Fluid Phase Equilib, 2007, 257: 27-34

Jacquemin J, Gomes GMC, Husson P, Majer VJ. Chem Thermodyn, 2006, 38: 490-502

Lee BC, Outcalt SL. J Chem Eng Data, 2006, 51: 892-897

Gomes GMC. J Chem Eng Data, 2007, 52: 472-475

Anderson JL, Dixon JK, Brennecke JF. Acc Chem Res, 2007, 40: 1208-1216

Florusse LJ, Raeissi S, Peters CJ. J Chem Eng Data, 2008, 53: 1283-1285

Carlisle TK., Bara JE, Gabriel CJ, Noble RD, Gin DL. Ind Eng Chem Res, 2008, 47: 7005-7012

Finotello A, Bara JE, Camper D, Noble RD. Ind Eng Chem Res, 2008, 47: 3453-3459

Zhang J, Zhang QH, Qian BT, Deng YQ. J Chem Eng Data, 2007, 52: 2277-2283

Palgunadi J, Hong SY, Lee JK, Jung S. Chem Eng Process, 2010, 49: 192-198

Lee JM, Palgunadi J, Kim JH, Jung S, Choi Y, Cheong M, Kim HS. Phys Chem Chem Phys, 2010, 12: 1812-1816 
Palgunadi J, Hong SY, Lee JK, Lee H, Lee SD, Cheong M, Kim HS. J Phys Chem B, 2011, 115: 1067-1074

Zhao X, Xing HB, Yang QW, Li RL, Su BG, Bao ZB, Yang YW, Ren QL. J Phys Chem B, 2012, 116: 3944-3953

Xing HB, Zhao X, Yang QW, Su BG, Bao ZB, Yang YW, Ren QL. Ind Eng Chem Res, 2013, 52: 9308-9316

Xing HB, Zhao X, Li RL, Yang QW, Su BG, Bao ZB, Yang YW, Ren QL. ACS Sustain Chem Eng, 2013, 1: 1357-1363

Anthony JL, Maginn EJ, Brennecke JF. J Phys Chem B, 2002, 106: 7315-7320

Yuan X, Zhang SJ, Chen YH, Lu XM, Dai WB, Mori R. J Chem Eng Data, 2006, 51: 645-647

Raeissi S, Peters CJ. Fluid Phase Equilib, 2010, 294: 67-71

Liu XY, Afzal W, He MG, Prausnitz JM. J Phys Chem B, 2013, 117: 10534-10539

Liu XY, Afzal W, Prausnitz JM. Ind Eng Chem Res, 2014, 53: 363-368

Liu XY, Afzal W, He MG, Prausnitz JM. AIChE J, 2014, 60: 2607-2612

Hert DG, Anderson JL, Aki SNVK, Brennecke JF. Chem Commun, 2005: 2603-2605

Cui XL, Chen KJ, Xing HB, Yang QW, Krishna R, Bao ZB, Wu H, Zhou W, Dong XL, Han Y, Li B, Ren QL, Zaworotko MJ, Chen BL.

Science, 2016, 353: 141-144

\title{
Separation of structurally-related compounds with ionic liquids
}

\author{
Wenbin Jin, Xuenan Li, Yi Zhang, Qiwei Yang, Huabin Xing", Qilong Ren \\ Key Laboratory of Biomass Chemical Engineering of Ministry of Education; College of Chemical and Biological Engineering, Zhejiang \\ University, Hangzhou 310027, China \\ *Corresponding author (email: xinghb@zju.edu.cn)
}

\begin{abstract}
As innovative media for separation, ionic liquids (ILs) have received significant attention in recent years. Especially, ILs have been successfully applied in the separations of structurally-related compounds due to their unique properties, such as multiple solvation interactions, and tunable structure and properties. Reduced environmental impact and improved separation efficiencies have been achieved using ILs compared to traditional separation mediums. In this review, the structural characteristics of ILs and the interactions between ILs and solute molecules at molecular level were systematically summarized. The recent progress involving separation of structurally-related compounds including natural bioactive products, organic compounds, and light hydrocarbons with ILs was introduced and summarized. Finally, the existing problems and the future research directions to address current challenges in the field of IL-based separation processes were discussed.
\end{abstract}

Keywords: ionic liquids, separation, bioactive compounds, light hydrocarbons, structurally-related compounds

doi: $10.1360 / \mathrm{N} 032016-00121$ 